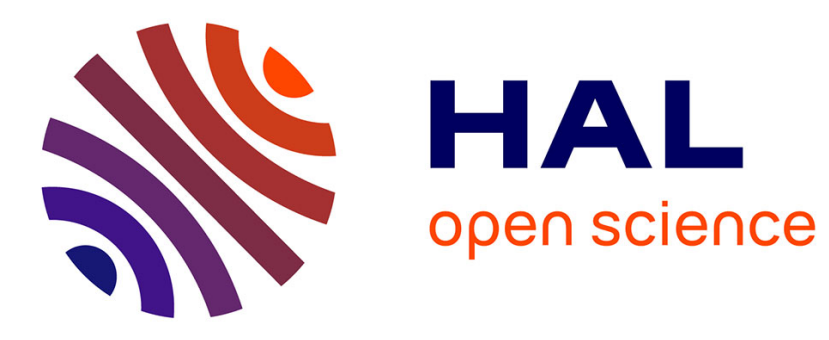

\title{
On Ontological Expressivity and Modelling Argumentation Schemes Using COGUI
}

Wael Hamdan, Rady Khazem, Ghaida Rebdawi, Madalina Croitoru, Alain Gutierrez, Patrice Buche

\section{- To cite this version:}

Wael Hamdan, Rady Khazem, Ghaida Rebdawi, Madalina Croitoru, Alain Gutierrez, et al.. On Ontological Expressivity and Modelling Argumentation Schemes Using COGUI. Thirty-fourth SGAI International Conference on Innovative Techniques and Applications of Artificial Intelligence, 2014, Cambridge, United Kingdom. pp.5-18, 10.1007/978-3-319-12069-0_1 . lirmm-01109928

\section{HAL Id: lirmm-01109928 https://hal-lirmm.ccsd.cnrs.fr/lirmm-01109928}

Submitted on 27 Jan 2015

HAL is a multi-disciplinary open access archive for the deposit and dissemination of scientific research documents, whether they are published or not. The documents may come from teaching and research institutions in France or abroad, or from public or private research centers.
L'archive ouverte pluridisciplinaire HAL, est destinée au dépôt et à la diffusion de documents scientifiques de niveau recherche, publiés ou non, émanant des établissements d'enseignement et de recherche français ou étrangers, des laboratoires publics ou privés. 


\title{
On Ontological Expressivity and Modelling Argumentation Schemes using COGUI
}

Wael Hamdan and Rady Khazem and Ghaida Rebdawi and Madalina Croitoru and Alain Gutierrez and Patrice Buche

\begin{abstract}
Knowledge elicitation, representation and reasoning explanation by / to non computing experts has always been considered as a crafty task due to difficulty of expressing logical statements by non logicians. In this paper, we use the COGUI editor in order to elicit and represent Argumentation Schemes within an inconsistent knowledge base. COGUI is a visual, graph based knowledge representation editor compatible with main Semantic Web languages. COGUI allows for default reasoning on top of ontologies. We investigate its use for modelling and reasoning using Argumentation Schemes and discuss the advantages of such representation. We show how this approach can be useful in the practical setting of EcoBioCap where the different Argumentation Schemes can be used to lead reasoning.
\end{abstract}

Key words: Applications, Argumentation, AIF, Argumentation Schemes, Reasoning, COGUI

\author{
Wael Hamdan \\ Higher Institute of Applied Science and Technology (HIAST), Syria \\ e-mail: waelHamdan1977@gmail.com \\ Rady Khazem \\ Higher Institute of Applied Science and Technology (HIAST), Syria \\ Ghaida Rebdawi \\ Higher Institute of Applied Science and Technology (HIAST), Syria \\ Madalina Croitoru \\ University Montpellier 2, France \\ e-mail: croitoru@lirmm.fr \\ Alain Gutierrez \\ University Montpellier 2, France \\ Patrice Buche \\ IATE, INRA, France \\ e-mail: patrice.buchedsupagro.inra.fr
}


W. Hamdan et al.

\section{Introduction}

COGUI $^{1}$ (COnceptual Graphs User Interface) is a knowledge base editor in which knowledge is encoded as graphs and that supports sound and complete graph based reasoning operations. The COGUI editor will allow to encode knowledge bases expressed in a logical formalism encompassing Semantic Web main languages: RDF/S, OWL and Datalog+ [12]. COGUI graphs have a semantics in first-order logic (FOL) and reasoning tasks operate directly on the knowledge defined by the user (the graphs) and not on their translation into logical formulas [5]. COGUI can import and export all major Semantic Web main languages (RDF/S, OWL, Datalog, CGIF, $\operatorname{Cog}$ XML) and has been recently extended to support non-monotonic reasoning using default rules. This extension was developed given the need induced by practical applications to support inconsistent ontology based reasoning([4], [2]).

Argumentation Schemes (AS) are used to classify forms of arguments people exchange in their daily life discourses. They are used to identify and evaluate common and stereotypical forms of arguments [13]. The Argument Interchange Format (AIF; [6]), largely based on AS, proposes an "unified Ontology" for argumentation. The first AIF Ontology was proposed by [10] and was based on Resource Description Framework Schema RDFS, this AIF-RDF Ontology was implemented in a Semantic Web-based system named ArgDF. This work was extended in [9] by introducing OWL-based AIF Ontology in Description Logic DL [1]. This ontology enabled automatic scheme classifications, instance classification, inference of indirect support in chained argument structures, and inference of critical questions. The model focused on typology and overall structure of the arguments and did not enable argument acceptability. Furthermore, the type of reasoning in AS is non-monotonic. The reasoning in this OWL Ontology is based on a subset of first order predicate logic thus non-monotonic reasoning is not supported (for more details please see the expressive overlaps among knowledge representation languages illustrated in [7]).

In this article we present an AIF compatible Ontology for modelling AS that extends the expressivity of the existing work to default rule base reasoning. The ontology is available in RDFS, OWL, Datalog+ or CogXML and has been built using COGUI. The model extends the various types of inference supported in [9] by supporting argument acceptability and enabling non-monotonic reasoning. We model the following AS: argument from expert opinion, argument from analogy, and argument from popular opinion. Our work distinguishes itself from the AIF Ontology not only by (1) the expressivity brought by default rules but also by (2) its practical application. Indeed, we model the various domain application statements (we will later explain this feature in more details) using logical facts. This distinguishes us from the model introduced by [9] which deals with statements as black-boxes and not logical facts made of grounded atoms one can reason upon. We will showcase next an example based on the COGUI to illustrate types of inference supported by our AIF Ontology and which are not supported in [9].

\footnotetext{
${ }^{1} \mathrm{http} / / /$ www.lirmm.fr/cogui/
} 
AIF Modelling in Cogui

\section{Motivating Example}

The "argument from position to know" [14] has the following elements:

- Position to know premise: $\mathrm{E}$ is in a position to know whether $\mathrm{A}$ is true (false).

- Assertion premise: $\mathrm{E}$ asserts that $\mathrm{A}$ is true (false).

- Conclusion: A may plausibly be taken to be true (false).

The scheme has a set of critical questions, we mention for example the trustworthiness question:" Is E reliable?".

Figure 1 shows an argument network for this scheme using the underlying ontology of ArgDF [9]. This follows a graph based depiction of RDF and namely the nodes of the network representing subjects or objects of the RDF triple while the edges are labelled with the predicate. According to RDF/S semantics two nodes $s$ and $o$ linked by an edge labelled with $p$ have the logical semantics of $p(s, o)$. In the case of Figure 1 the nodes can represent either domain statements such as: "Brazil is the best football team in the world" or generic arguments such as: " $\mathrm{E}$ is in position to know whether A is true or false". This means that we cannot reason further about Brazil being the best football team in the world (for instance inferring that Brazil won the World Cup). The statements are seen as black-boxes and we cannot reason about the knowledge contained in the black-boxes.

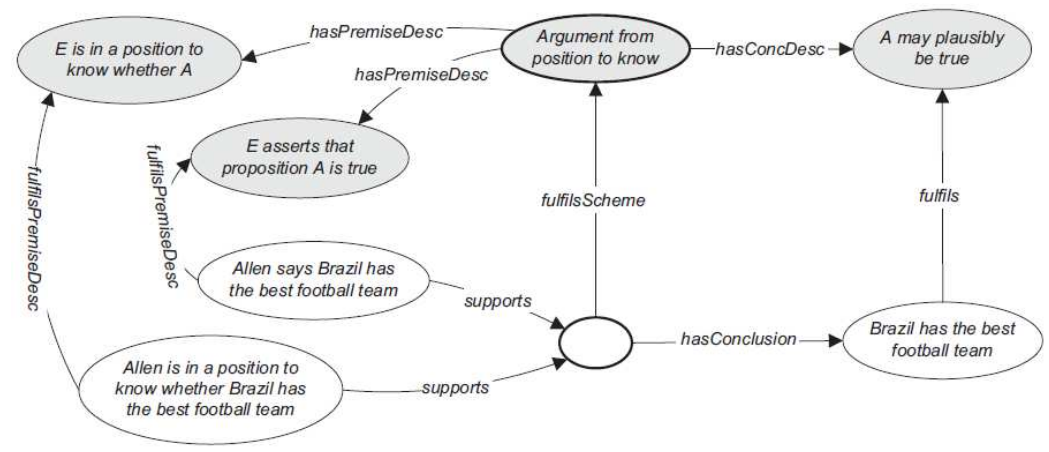

Fig. 1: "Argument from position to know" using ArgDF Ontology

Rahwan et al [9] proposed ontology does reasoning in order to retrieve argument instances semantically. More precisely, in this particular example, the necessary and sufficient conditions for an instance to be classified as an argument from position to know are as follows:

ArgFromPositionToKnow(PresumptiveArgument $\bigcap$

$\exists$ hasConclusion.KnowledgePositionStmnt $\bigcap$

$\exists$ hasPremise.PositionToHaveKnowledgeStmnt $\bigcap$

$\exists$ hasPremise.KnowledgeAssertionStmnt) 
Our proposed model (illustrated in Figure 2) models all the grounded atoms as different pieces of knowledge, practically splitting the black-boxes of the previous model. This means that when we apply the rule of inference associated with this scheme we do not loose any expressivity as per existing work. However we additionally model the exceptions, and link them directly to the premises of the argument. In this example we link "LackOfReliabilityStmnt" to "Allen". Exceptions in work of Rahwan are not treated in a non monotonic manner but they are pure syntactic flags not handled by any reasoning engine. This type of reasoning is supported in our model using the default rule associated with the scheme.

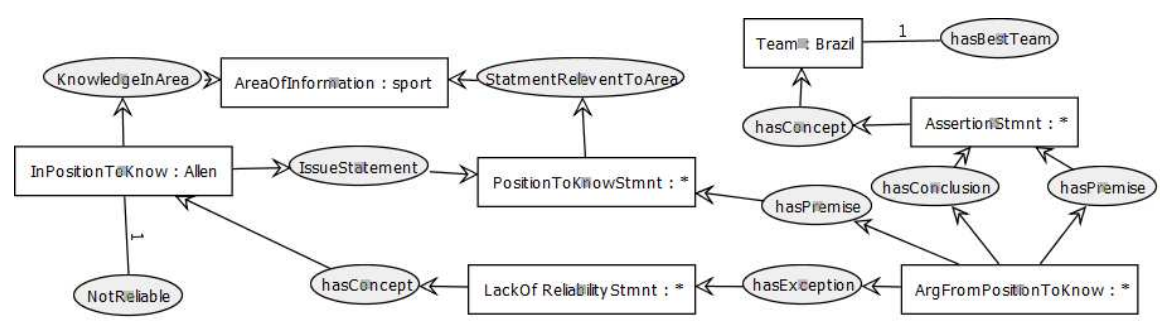

Fig. 2: "Argument from position to know" using our Model

Furthermore, suppose we have another person "Martin", also "in position to know" who says the opposite: i.e "Brazil is not the best team". The model of Rahwan will not capture the conflict unless we explicitly state (and thus not infer) that there is a conflict between theses two arguments. In our model, and thanks to the rule defined in Figure 6a, we conclude that the conclusion statements of the two arguments (issued by "Allen" and "Martin") are contradictory. Thereafter, the rule (depicted in Figure 5) will infer the conflict scheme and the attack relations.

\section{Background Notions}

\subsection{AIF Ontology}

The AIF model was introduced by the research community to represent a consensus "abstract model" for argumentation in order to facilitate the exchange of semistructured arguments among different argumentation systems. [10] and [9] illustrated the use of the proposed abstract model in argumentations systems by introducing concrete realizations. The AIF model is based on AS, and each AS in AIF has a name, set of premises, conclusion and a set of predefined critical questions. Critical questions are used to identify potential weaknesses of the argument and thus possibilities for the proponent to "attack" this argument. 


\subsection{Conceptual Graphs Knowledge Bases and Cogui}

The Conceptual Graphs (CGs) formalism introduced by [12] and [8] is a knowledge representation and reasoning formalism representing a subset of first order logic compatible with the major Semantic Web languages. A CGs knowledge base comprises the vocabulary part (also called support) that represents the domain ontology (equivalently the TBox in Description Logics, Datalog rules or RDF/S schema), and the assertions part called basic CGs (BCGs) that represents facts or assertions (ABox, DB instances, RDF files etc.). The vocabulary is composed of two partially ordered sets by a specialization relation: a set of concepts and a set of relations of any arity (the arity is the number of arguments of the relation). The specialization relation is defined as: $x$ is a specialization of $X$, if $x$ and $X$ are concepts, specialisation means that every instance (individual) of the concept $\mathrm{x}$ is also an instance of the concept X. A basic conceptual graph (BG) is a bipartite graph composed of: (i) a set of concept nodes, represents entities; (ii) a set of relation nodes, represents relationships between these entities or properties of them; (iii) a set of edges linking relation nodes to concept nodes. A concept node is labeled by a couple $t: m$ where $t$ is a concept (and more generally, a list of concepts) called the type of the node, and $\mathrm{m}$ is called the marker of this node: this marker is either the generic marker, denoted by $*$, if the node refers to an unspecified entity, otherwise this marker is a specific individual name.

The CGs model comprises also more complex constructs such as complex first order rules (equivalent to tuple generating dependencies in databases or Datalog+ rules) and default rules (which allow for non-monotonic reasoning).

Rules: a rule expresses implicit knowledge of the form "if hypothesis then conclusion", where hypothesis and conclusion are both basic graphs. This knowledge can be made explicit by applying the rule to a specific fact: intuitively, when the hypothesis graph is found in a fact, then the conclusion graph is added to this fact.

Default rules: CGs default rules are based on Reiters default logics in [11] and [3]. They are defined by a tuple $D R=\left(H, C, J_{1} \ldots J_{k}\right)$, where $H$ is called the hypothesis, $C$ the conclusion and $J_{1}, \ldots, J_{k}$ are called justifications of the default. All components of $D R$ are themselves basic CGs. The intuitive meaning of a CG default is : if $H$ holds for all individuals, then $C$ can be inferred, provided that no justification $J_{i}$ (for all $i$ from 1 to $k$ ) holds.

Negation in Conceptual Graphs is represented by the means of the negative constrains which are basic graphs with the semantic that if they occur in the knowledge base the knowledge base is inconsistent. Please note that this specific kind of negation is equivalent to the negation used by the OWL and Description Logics as well as the integrity constrains used by databases. We can also impose positive constraints, that is, pieces of knowledge that need to appear in the graph (and the fact that it does not appear renders the graph inconsistent). Both constraints will be used later on for modelling purposes.

In the following section let us introduce the basic ontology which will include the support of the CGs, rules of inference and constraints. 
W. Hamdan et al.

\section{AIF Ontology for Argumentation Schemes}

The backbone of the AIF Ontology is shown in Figure 3 and follows the model of the ontology defined in [9]. The hierarchy of concepts includes on the top level: Statements that describe statements which could be issued, Schemes which describe arguments made up of statements. Three type of schemes are defined. The first scheme is the rule scheme that defines types of arguments that could by defined. The second is a conflict scheme that represents the attack relations between arguments. The third is the preference scheme and it includes logical and presumptive preference. The relation types defined between arguments in ruleScheme and Statements are: hasPremise, hasConclusion and hasException. These relations denote that an argument may have premises, conclusions and exceptions respectively. A statement could attack a conflictScheme through the relation attack and could be attacked by this scheme with the relation conflictAttacked. Other relation types denote the facts that a statement may be true (isTrue) or false (isFalse).

After defining the backbone of concepts and relations, we need to impose the con-

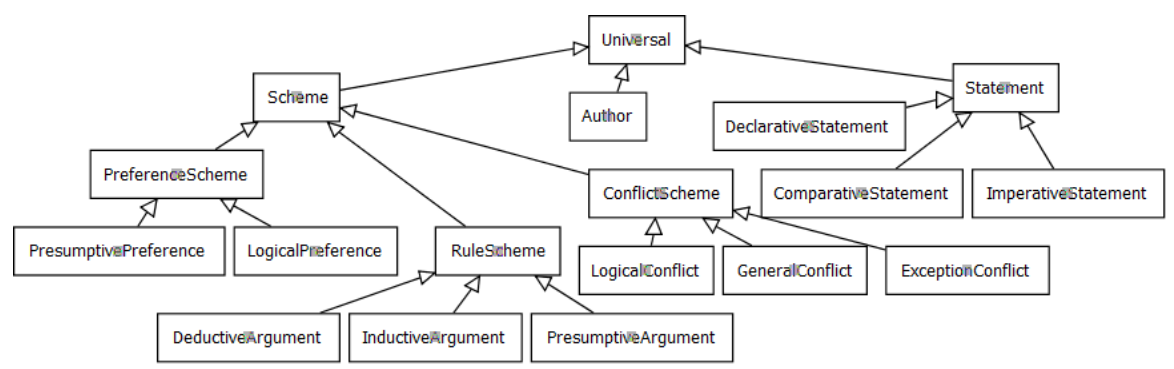

(a) Concept types

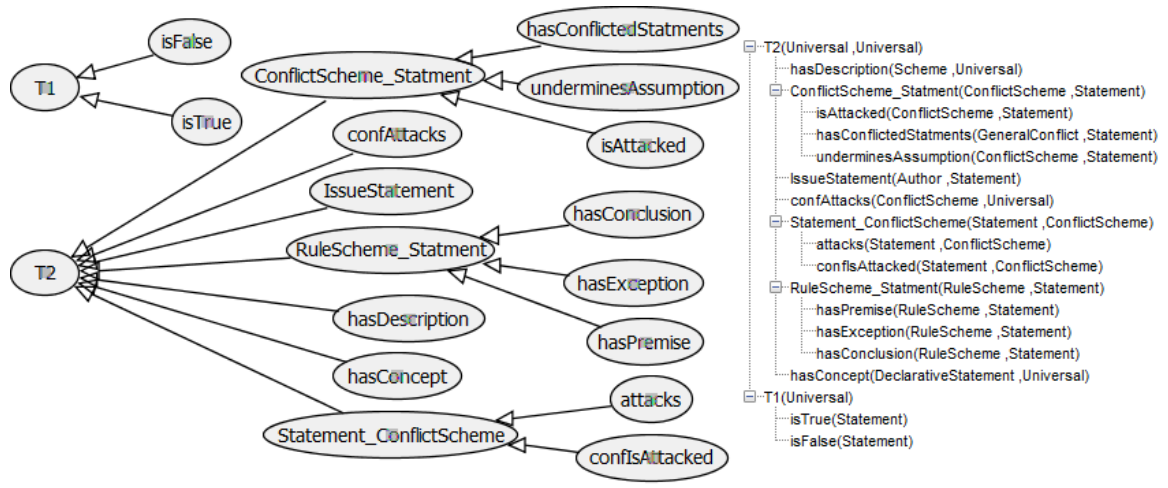

(b) Relation types

Fig. 3: AIF Ontology

straints to ensure model consistency. We use a positive constraint to say that every 
argument has at least one premise and one conclusion as illustrated in Figure 4a. In order to ensure that every argument has at most one conclusion we use a negative constraint as shown in Figure 4b.

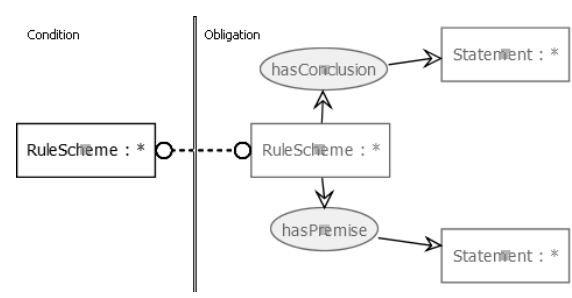

(a) Positive Constraint

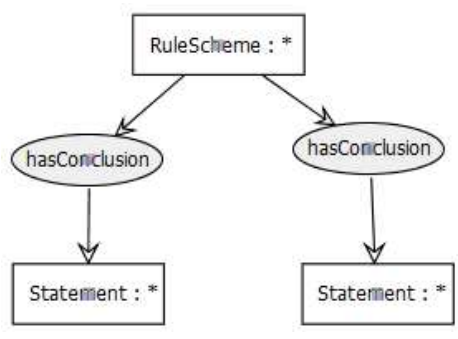

(b) Negative Constraints

Fig. 4: Conclusion Constraints

Attacks among Arguments: Specializations of the concept conflictScheme are used to represent attacks among different arguments, GeneralConflict instances capture simple symmetric and asymmetric attacks among arguments, while ExceptionConflict instances represent exceptions to rules of inference.

We first define the rule for the symmetric attack between two arguments as follows: if two statements $S_{1}, S_{2}$ belong to the concept GeneralConflict through the relation hasConflictedStatments(GeneralConflict, Statement), i.e there is a conflict between these two statements, and if one statement appears in the conclusion of argument $\mathrm{A}$ and the other appears in the conclusion of argument B, we say that there is a symmetric attack between the arguments A and B. Figure 5 illustrates the symmetric conflict rule. The rule semantics is as follows: if $\forall S_{1}, S_{2} \in$ Statement, $\exists G F \in$ GeneralConflict, such that hasConflictedStatments $\left(G F, S_{1}\right)$, hasConflictedStatments $\left(G F, S_{2}\right)$ and if $\exists A_{1}, A_{2} \in$ RuleScheme, such that

hasConclusion $\left(A_{1}, S_{1}\right)$ and hasConclusion $\left(A_{2}, S_{2}\right)$ then there is a symmetric conflict defined by the relations: confAttacks, confAttacked, attacks and isAttacked.

In addition to the general conflict rule defined above, we define in Figure 6a the rule that models the relation hasConflictedStatments (GeneralConflict, Statement) as follows: when a statement $S$ is plausible to be evaluated "true" and "false" at the same time, then the two instances of $S: S_{1}$ (evaluated "true") and $S_{2}$ (evaluated "false") belong to the relation hasConflictedStatments. Thus, having $S_{1}, S_{2}$ belong to the relation hasConflictedStatments and using the previous rule we conclude that if $S_{1}$ and $S_{2}$ appear in the conclusions of two arguments $\mathrm{A}$ and $\mathrm{B}$, then there will be a symmetric attack between $\mathrm{A}$ and $\mathrm{B}$.

The second type of conflict is the exception conflict (please see Figure 6b), and this is the case when the statement is the conclusion of an argument A and at the same time the exception of other argument B. In this case the conclusion of the argument A attacks the argument B through the concept ExceptionConflict. 


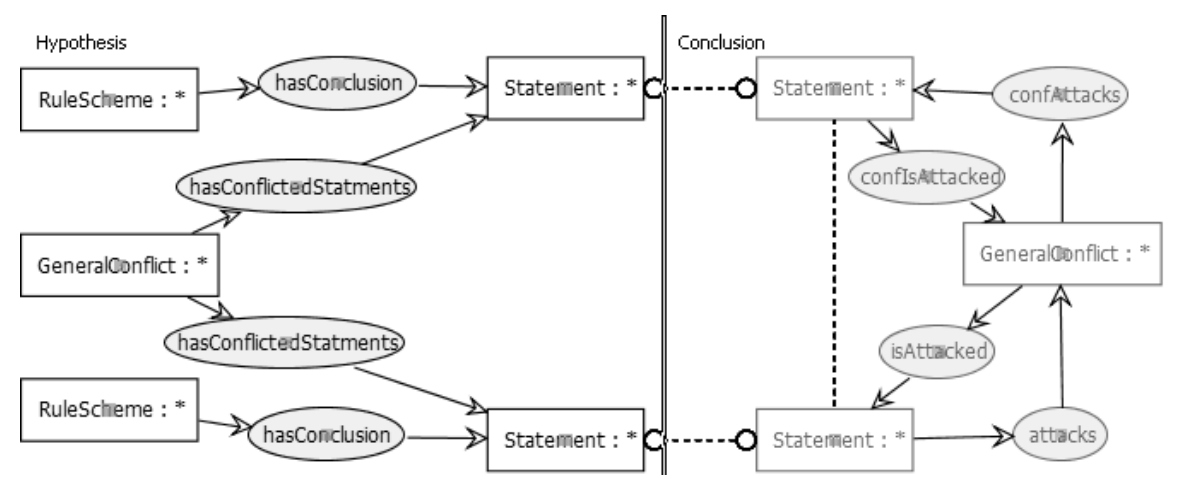

Fig. 5: Symmetric Conflict Rule

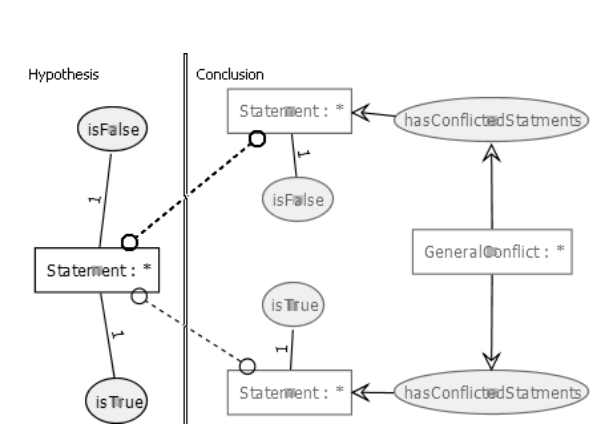

(a) Statement Contradiction

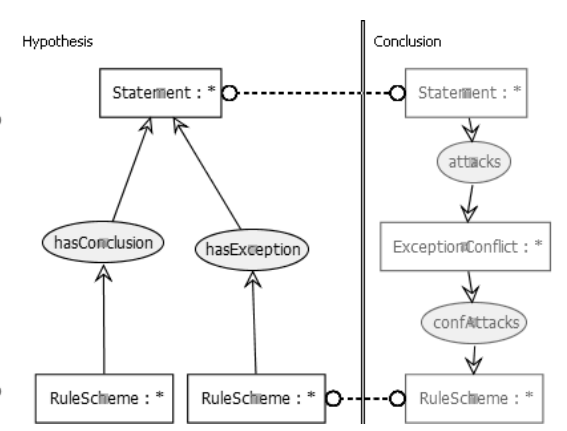

(b) Except Conflict Rule

Fig. 6: Conflict Constraints

\section{Modelling Argumentation Schemes}

Our methodology for modelling new AS includes the following three steps:

- STEP 1 - Ontology enriching. We enrich the vocabulary of our AIF Ontology with new concepts and relation types, add a concept that represent the new scheme as descendent of rule scheme and add concepts according to critical questions.

- STEP 2 - Rules definition. We define the rules of inference that will enable AS inference (i.e identifying the schemes) and critical questions inference.

- STEP 3 - Default rules definition. We introduce the default rules that will define the non-monotonic reasoning. The generic inference rule used is defined as follows: "If an argument conforms to an AS, we conclude that its conclusion is true, unless one of its exceptions holds".

Let us now explain how we apply this methodology in order to define three Argumentations Schemes. 
AIF Modelling in Cogui

\section{COGUI Implementation of Argumentation Schemes}

\subsection{Argument from Expert Opinion}

In many forms of arguments people cite an authority to back up their point of view. In other words they indicate that someone (the authority cited) could give reasons that back up the statements they defend. This form of arguments is called "appeal to authority" or "argument from position to know". Depending on the type of the cited authority, the AS would be "Argument from Expert Opinion" if the authority is an expert, or "Argument from Witness Testimony", if the person is a witness in the situation at hand. This type of arguments comes with common critical questions such as questioning the reliability of the authority, more precisely: is the cited person an authority? or is the authority an authority in the domain under discussion?

We will model the scheme "argument from expert opinion" as a sub-scheme of the scheme "argument from position to know" (for the lack of space, we will not present the full modeling of "argument from position to know", and only a part of it is depicted in the motivating example). The Scheme [14] has the following elements:

- Expertise premise: The source $\mathrm{E}$ is an expert in the domain D that is containing proposition A.

- Assertion premise: $\mathrm{E}$ asserts that $\mathrm{A}$ is true (false).

- Conclusion: A may plausibly be taken to be true (false).

Critical questions are:

1. Expertise: How credible is expert E?

2. Trustworthiness: Is E reliable?

3. Consistency: Is A consistent with the testimony of other experts?

4. Backup evidence: Is A supported by evidence?

We model this scheme as follows:

- STEP 1 - Ontology enriching. We add a concept named ArgFromExpertOpinion to represent the scheme as descendent of rule scheme. In order to model the expertise premise, we add the concept ExpertStmnt as descendent of

DeclarativeStatement. This statement is translated as an Expert $\mathrm{E}$ issues a Statement through the relation IssueStatement, and the issued statement belongs to a domainOfExperience in which $\mathrm{E}$ has enough expertise. The statement AssertionStmnt denotes that E asserts that A is true (false) which is the assertion premise, and also denotes the conclusion of the argument which is $\mathrm{A}$ may plausibly be taken to be true (false).

- STEP 2 - Rules definition. The rule could be written as: "if a rule scheme $R$ has a premise of type ExpertStmnt and has a premise of type AssertionStmnt which is also its conclusion then $R$ belongs to ArgFromExpertOpinion". This is formally depicted in Figure 7, 
- STEP 3 - Default rules definition. The default rule is formulated as: "If a statement $S$ is issued by an expert $E$ in the domain of the statement $S$, we conclude that $S$ is true, unless for example the expert $E$ was not reliable or inconsistent with other experts". Figure 8 depicts this rule.

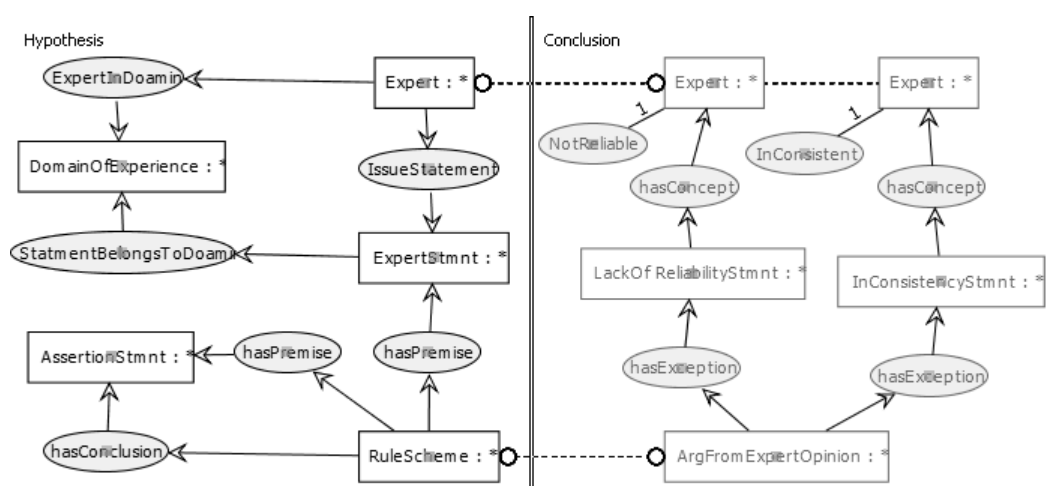

Fig. 7: Expert Rule

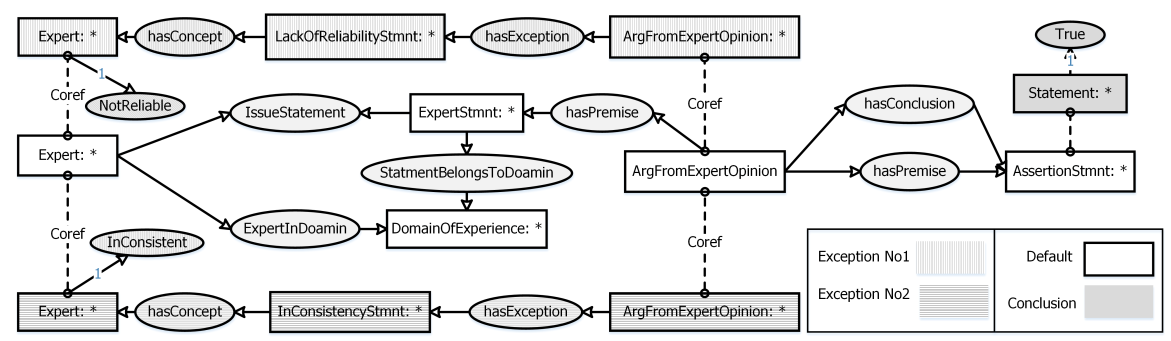

Fig. 8: Expert Default Rule

\subsection{Argument from Analogy}

The AS for argument from analogy can be represented as follows (please see [15] for more details): 
AIF Modelling in Cogui

- Similarity Premise: Generally, case $\mathrm{C} 1$ is similar to case $\mathrm{C} 2$

- Base Premise: A is true (false) in case C1.

- Conclusion: A is true (false) in case $\mathrm{C} 2$.

The critical questions which are:

1. Are $\mathrm{C} 1$ and $\mathrm{C} 2$ similar in the respect cited?

2. Are there differences between $\mathrm{C} 1$ and $\mathrm{C} 2$ that would tend to undermine the force of the similarity cited?

3. Is there some other case $\mathrm{C} 3$ that is also similar to $\mathrm{C} 1$, where $\mathrm{A}$ is false (true)?

The COGUI methodology to implement this scheme is as follows:

- STEP 1 - Ontology enriching. Since we consider two similar cases $C_{1}, C_{2}$, we obviously need to add the concept Case and the relation isSimilarTo. We also refer to the fact that a statement $S$ is true in case $C$ by stating that: $S$ is issued by the Case $C$. Thus, the argument could be written as follows: if a statement $S$ is issued by a case $C_{1}$, and if $C_{2}$ is similar to $C_{1}$, then $S$ will be also considered as issued by the case $C_{2}$. We finally need to add the concepts NotSimilarInRespectCited, UnderminingDifferences, and ExistSimilarCaseWithDifferentResult for representing the three critical questions respectively.

- STEP 2 - Rules definition. The rule could be written as: "if a rule scheme $R$ has first premise: statement $S$ is issued by case $C_{1}$, and if $R$ has a second premise: there exists a case $C_{2}$ which is similar to $C_{1}$, and has a conclusion: the statement $S$ is is also issued by case $C_{2}$, then the scheme $R$ belongs to ArgFromAnalogy". This is formally depicted in Figure 9.

- STEP 3 - Default rules definition. The default rule for this scheme could be formulated as: "if a statement $S$ is is issued by a Case $C_{1}$ and if exists a case $C_{2}$ which is similar to $C_{1}$, then we conclude that $S$ is true in case $C_{2}$, unless the similarity was not in the respect cited, or there were undermining differences, or if there exists a case $C_{3}$ which is similar to $C_{1}$ and in which $S$ is not true (false)". Figure 10 depicts a COGUI modelling of this rule (in order to have a readable and less complicated diagram we consider only two of the exceptions: NotSimilarInRespectCited and ExistSimilarCaseWithDifferentResult .

\subsection{Argument from Popular Opinion}

The argument from popular opinion as described by [14] is: If a large majority (everyone, nearly everyone, etc.) accepts A as true, this would be evidence that A is generally accepted. The structure of the scheme include the following elements:

- General acceptance premise: A is generally accepted as true.

- Presumption premise: If $\mathrm{A}$ is generally accepted as true, it gives reason for A.

- Conclusion: There is a reason in favor of A. 
W. Hamdan et al.

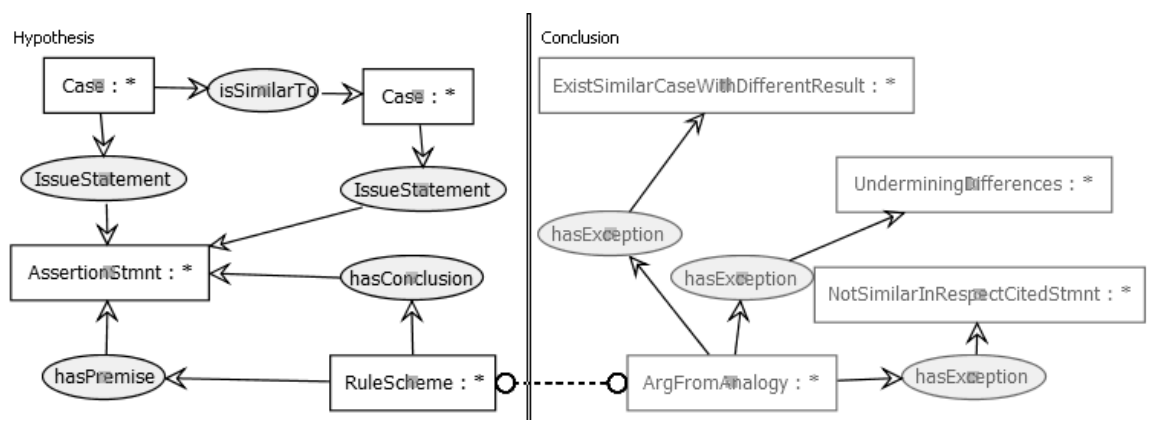

Fig. 9: Analogy Rule

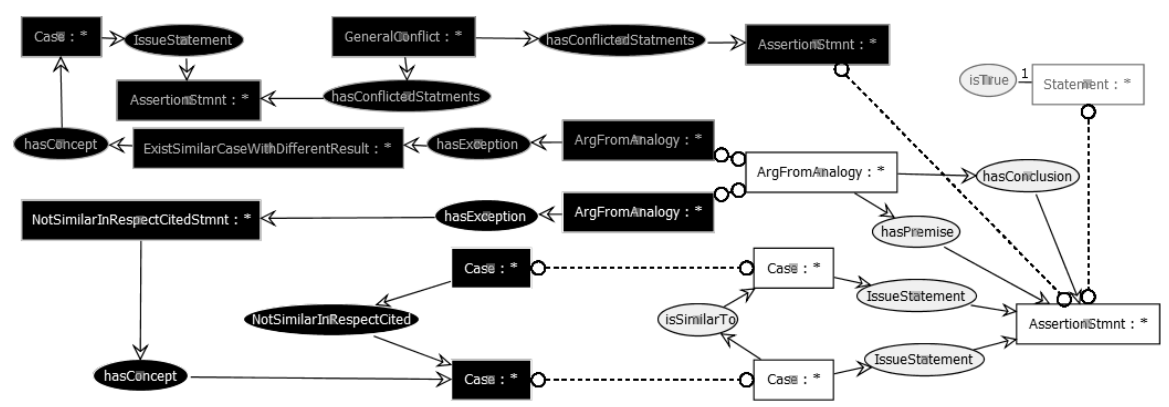

Fig. 10: Analogy Default Rule

The following two critical questions match the scheme:

1. What evidence, such an appeal to common knowledge, supports the claim that A is generally accepted as true?

2. Even if A is generally accepted as true, are there any good reasons for doubting that it is true?

The COGUI methodology to represent this scheme is as follows:

- STEP 1 - Ontology enriching. We refer to the fact that the statement $S$ is generally accepted by public opinion by stating that: $S$ is issued by PublicOpinion. Therefore, the statement $S$ is the premise and the conclusion of the rule scheme. Consequently we enrich our ontology with the concepts ArgFromPublicOpinion and PublicOpinion. We need also to add the concept LackOfEvidence and the relation issuedWithLackOfEvedence(statment) for the first critical question, and the concept ExistGoodReasonsForDoubting and relation

hasGoodReasonsForDoubting (statment) for the second critical question.

- STEP 2 - Rules definition. The rule could be written as: "if a statement $S$ is issued by a PublicOpinion and if $S$ is the premise and the conclusion of a rule scheme $R$, then $R$ belongs to ArgFromPublicOpinion". This is formally depicted in Figure 11. 
- STEP 3 - Default rules definition. The default rule for this scheme could be formulated as: "if a statement $S$ is issued by a PublicOpinion, we conclude that $S$ is true, unless there is a lack of evidences or there is good reasons for doubting". Figure 12 includes a modeling of this rule.

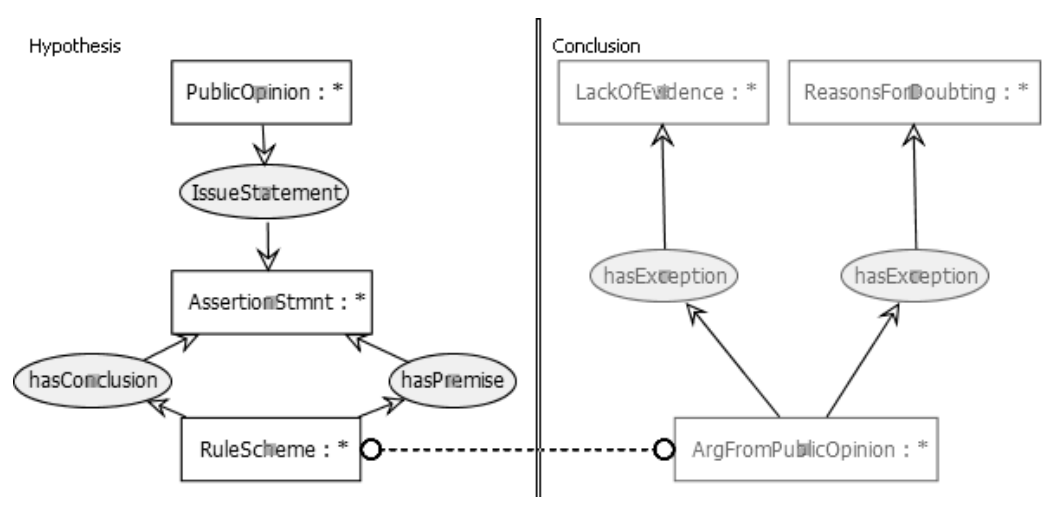

Fig. 11: Public Opinion Rule

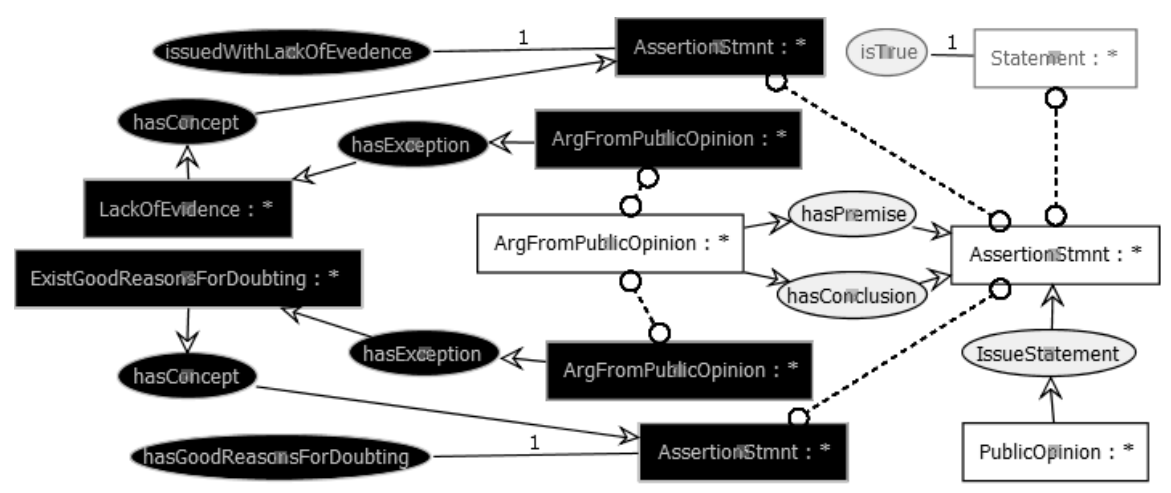

Fig. 12: Public Opinion Default Rule

\section{Conclusions}

This paper presented a methodology and the modelling of AS using COGUI (graphical knowledge modelling and querying environment) to represent and use knowledge about arguments. This approach showed how a generally useful and accessible 
tool can be used to do non-monotonic reasoning which could be characterized as represent common knowledge. Our work distinguishes itself from the state of the art due to the expressiveness of the rules (that allow for non monotonic reasoning) as well as the fine grain representation of arguments (as opposed to black-box approaches taken by previous work). The ontology is publicly available and we are currently investigating its use for reasoning about decision making in agri food scenarios. This is an ongoing ontological modelling effort and we are currently extending our work with other schemes in the literature.

Acknowledgment: The research leading to these results has received funding from the European Community's Seventh Framework Programme (FP7/ 2007-2013) under the grant agreement no FP7-265669-EcoBioCAP project.

\section{References}

1. F. Baader, D. Calvanese, D. L. McGuinness, D. Nardi, and P. F. Patel-Schneider, editors. The Description Logic Handbook: Theory, Implementation, and Applications. Cambridge University Press, 2003.

2. J.-F. Baget, M. Croitoru, J. Fortin, and R. Thomopoulos. Default conceptual graph rules: Preliminary results for an agronomy application. In ICCS, pages 86-99, 2009.

3. H. Boley, M. Kifer, P.-L. Patranjan, and A. Polleres. Rule interchange on the web. In Reasoning Web, pages 269-309, 2007.

4. P. Buche, V. Cucheval, A. Diattara, J. Fortin, and A. Gutierrez. Implementation of a knowledge representation and reasoning tool using default rules for a decision support system in agronomy applications. In GKR, pages 1-12, 2013.

5. M. Chein, M.-L. Mugnier, and M. Croitoru. Visual reasoning with graph-based mechanisms: the good, the better and the best. Knowledge Eng. Review, 28(3):249-271, 2013.

6. C. I. Chesñevar, J. McGinnis, S. Modgil, I. Rahwan, C. Reed, G. R. Simari, M. South, G. Vreeswijk, and S. Willmott. Towards an argument interchange format. Knowledge Eng. Review, 21(4):293-316, 2006.

7. B. N. Grosof, I. Horrocks, R. Volz, and S. Decker. Description logic programs: combining logic programs with description logic. In $W W W$, pages 48-57, 2003.

8. M.-L. Mugnier and M. Chein. Graph-based knowledge representation. Advanced Information and Knowledge Processing. Springer, London, 2009.

9. I. Rahwan, B. Banihashemi, C. Reed, D. Walton, and S. Abdallah. Representing and classifying arguments on the semantic web. Knowledge Eng. Review, 26(4):487-511, 2011.

10. I. Rahwan, F. Zablith, and C. Reed. Laying the foundations for a world wide argument web. Artif. Intell., 171(10-15):897-921, 2007.

11. R. Reiter. A logic for default reasoning. Artificial intelligence, 13(1):81-132, 1980.

12. J. F. Sowa. Conceptual Structures: Information Processing in Mind and Machine. AddisonWesley, 1984.

13. D. Walton. Argumentation schemes for presumptive reasoning. Psychology Press, 1996.

14. D. Walton. Fundamentals of critical argumentation. Cambridge University Press, 2006.

15. D. Walton, C. Reed, and F. Macagno. Argumentation Schemes. Cambridge University Press, 2008. 